\title{
Is lower tourniquet pressure during total knee arthroplasty effective? A prospective randomized controlled trial
}

\author{
Tae Kyun Kim, Ankur B. Bamne ${ }^{2}$, Jae Ang $\mathrm{Sim}^{3}$, Ji Hyeon Park ${ }^{3}$ and Young Gon Na ${ }^{4,5^{*}}$ (D)
}

\begin{abstract}
Background: Higher tourniquet pressures may be associated with an increased risk of complications. We aimed to determine (1) whether a lower tourniquet pressure [systolic blood pressure (SBP) $+120 \mathrm{mmHg}$ ] is as effective as conventional tourniquet pressure (SBP $+150 \mathrm{mmHg}$ ) in providing a bloodless surgical field and decreasing blood loss, and (2) whether lowering the tourniquet pressure decreases tourniquet-related complications compared to conventional inflation pressure.

Methods: One hundred and sixty knees in 124 patients undergoing total knee arthroplasty (TKA) were randomly allocated to either conventional $(n=80)$ or lower inflation pressure group $(n=80)$. The quality of the initial surgical field and occurrence of intraoperative blood oozing, hemoglobin drop, drained volume and calculated blood loss were assessed as efficacy variables. Safety outcome variables included post-operative pain, tourniquet site skin problems (ecchymosis, bullae, skin necrosis), and other tourniquet-related complications such as nerve palsy, venous thromboembolism, and delayed rehabilitation.
\end{abstract}

Results: A comparable bloodless surgical field was successfully provided in both groups (100\% vs. 99\%, $p=1.000$ ). One case in the conventional pressure group and two cases in the lower pressure group showed intraoperative blood oozing ( $p=1.000)$, which was successfully controlled after an increase of $30 \mathrm{mmHg}$ in the tourniquet inflation pressure. There was no difference in the hemoglobin drop, drained volume, and calculated blood loss. The two groups did not differ in any safety outcomes such as post-operative pain, thigh complications, and other tourniquet related complications.

Conclusion: This study demonstrates that a tourniquet inflation pressure of $120 \mathrm{mmHg}$ above the SBP is effective method during TKA.

Trial registration: The trial was with ClinicalTrials.gov (NCT01993758) on November 25, 2013.

Keywords: Knee, Arthroplasty, Tourniquet, Inflation pressure, Complication

\section{Background}

A tourniquet is commonly used during total knee arthroplasty (TKA) to have a clear bloodless field, which potentially reduces operative time, decreases intra-operative

\footnotetext{
* Correspondence: orthonyg@gmail.com

This work was performed at the Joint Reconstruction Center, Seoul National University Bundang Hospital (166 Gumi-ro, Bundang-gu, Seongnam-si, Gyeonggi-do (13620), Republic of Korea).

${ }^{4}$ Department of Orthopedic Surgery, CM Hospital, 13, Yeongdeungpo-ro 36-gil, Yeongdeungpo-gu, Seoul 07301, Republic of Korea

${ }^{5}$ Former affiliation: Gachon University Gil Medical Center, Incheon, Republic of Korea

Full list of author information is available at the end of the article
}

blood loss, and better prepares the cement-bone interface, despite the possible adverse effects associated with its use $[1,2]$. Several aspects related to tourniquet use remain debatable; one among them is the pressure used to inflate the tourniquet. An optimal tourniquet pressure should be determined to balance safety and efficacy. A higher tourniquet pressure ensures the reliable function of the tourniquet; however, it may lead to a greater incidence of complications [3]. These complications are commonly related to both the duration of its use and the inflation pressure. While a lower tourniquet pressure is safer [4], it may not provide a bloodless operative field. However, no

(c) The Author(s). 2019 Open Access This article is distributed under the terms of the Creative Commons Attribution 4.0 International License (http//creativecommons.org/licenses/by/4.0/), which permits unrestricted use, distribution, and reproduction in any medium, provided you give appropriate credit to the original author(s) and the source, provide a link to the Creative Commons license, and indicate if changes were made. The Creative Commons Public Domain Dedication waiver (http://creativecommons.org/publicdomain/zero/1.0/) applies to the data made available in this article, unless otherwise stated. 
consensus exists regarding the optimal target inflation pressure among orthopedic surgeons [5].

The pressure used to inflate the tourniquet varies among surgeons. While some prefer a uniform tourniquet inflation pressure for all patients [6-8], others vary it based on the systolic blood pressure (SBP) [9-11]. The prior approach does not take the SBP into account and may result in the use of higher tourniquet pressures. On the other hand, the reported safe margin added to the SBP ranges widely, from 100 to $250 \mathrm{mmHg}$ in the literature [12-15]. In our institution, adding $150 \mathrm{mmHg}$ to the patient's SBP was the routine method for determining the tourniquet inflation pressure; therefore, the mean inflation pressure was far lower than the fixed high tourniquet pressure such as 300 or $350 \mathrm{mmHg}$. Considering that higher tourniquet pressures are a risk factor for tourniquet-related complications, we sought to further reduce the tourniquet inflation pressure from our conventional method, SBP $+150 \mathrm{mmHg}$. Although there were studies which determined the tourniquet pressure as SBP $+100 \mathrm{mmHg}$ [14-16], a report of $17 \%$ of failure of achieving bloodless surgical field after sharp SBP rise seemed to be not acceptable to the authors [17]. Thus, we set the target amount of reduction in the tourniquet inflation pressure as $30 \mathrm{mmHg}$ rather than $50 \mathrm{mmHg}$.

We aimed to determine whether a lower tourniquet pressure [systolic blood pressure (SBP) $+120 \mathrm{mmHg}$ ] is as effective as conventional tourniquet pressure (SBP +150 $\mathrm{mmHg}$ ) in providing a bloodless surgical field and decreasing blood loss We also questioned whether lowering the tourniquet pressure can decrease tourniquet-related complications compared to conventional inflation pressure. We hypothesized that lower tourniquet pressures may achieve a bloodless surgical field similar to that achieved with conventional inflation pressure and present comparable blood loss, and that it may lead to a decrease in tourniquet-related complications.

\section{Methods}

\section{Study design}

This study was a single-center, double-blinded, randomized controlled trial). The sample size was calculated based on the difference in the primary outcome, namely the failure rate of the bloodless field. In previous literature, an incidence rate of $17 \%$ has been reported for the failure of achieving a bloodless field when the tourniquet pressure was determined to be $100 \mathrm{mmHg}$ above the SBP [17]. We borrowed this result for the lower tourniquet pressure group $(\mathrm{SBP}+120 \mathrm{mmHg})$ in our study, because no other data are currently available for this pressure. In addition, we experienced a $2 \%$ failure rate in the conventional pressure group $(\mathrm{SBP}+150 \mathrm{mmHg})$ in the pilot study. Seventy-three subjects were required in each group to detect $15 \%$ of the difference in the failure rate when the power was set at 0.8 , and the alpha value was set at 0.05 . Accounting for a dropout rate of $10 \%$, a total of 160 consecutive knees were enrolled in this study ( 80 in each group). This study was approved by the institutional review board of our institution. Written informed consent was obtained from all the participants in this study. This study was registered at www.clinicaltrials.gov (NCT01993758).

Patients scheduled to undergo primary TKA (unilateral or staged bilateral TKA) for advanced knee osteoarthritis were considered eligible for inclusion. We assessed 168 knees in 130 patients undergoing unilateral or staged bilateral primary TKA between November 2013 and March 2014 for eligibility. From these patients, we excluded six patients who fulfilled at least one of the following exclusion criteria: (1) SBP measured in the ward $>200 \mathrm{mmHg}$ $(n=0)$; (2) Thigh circumference $>78 \mathrm{~cm} \quad(n=0)$; (3) Anesthesia other than spinal anesthesia $(n=6) ;(4)$ peripheral vascular disease $(n=0)$; (5) refusal to participate in this study $(n=2)[4,7]$. The remaining 160 knees in 124 patients were randomly allocated to the conventional tourniquet pressure group ( $\mathrm{SBP}+150 \mathrm{mmHg}, n=80)$ or the lower tourniquet pressure group $(\mathrm{SBP}+120$ $\mathrm{mmHg}, n=80$ ) using a computer-generated randomized Table. A randomization table was created by an independent statistician, which had permuted blocks of four and six. No patients were excluded for any reason after allocation; therefore, 80 knees remained in each group in the final analysis (Fig. 1). The patients, operator and an independent investigator who collected all the information prospectively remained blind to the randomization until final data analysis. Demographic characteristics, thigh circumference, the prevalence of hypertension, diagnosis, preoperative hemoglobin, operated side, operation type, used implant, and the operation method were not different between the two groups (Table 1).

\section{Perioperative management and surgical technique}

A femoral nerve block was conducted preoperatively in the operating room. A bolus dose of $30 \mathrm{~mL}$ of $0.375 \%$ ropivacaine was administered through an indwelling catheter, followed by a continuous infusion of $0.2 \%$ ropivacaine, and the catheter was kept remained until the third post-operative day. Spinal anesthesia was used in all the patients with $10-15 \mathrm{mg}$ of $0.5 \%$ bupivacaine and $20 \mu \mathrm{g}$ of fentanyl.

Tourniquet was applied in all patients with identical manner. A layer of elastic stockinet and three layers of cotton padding were used under the tourniquet cuff [18]. The extremity was exsanguinated with an Esmarch wrap, and the automated pneumatic tourniquet (ATS 2000, Zimmer Inc., Warsaw, IN, USA) was inflated. Based on the allocation of the subjects, the inflation 


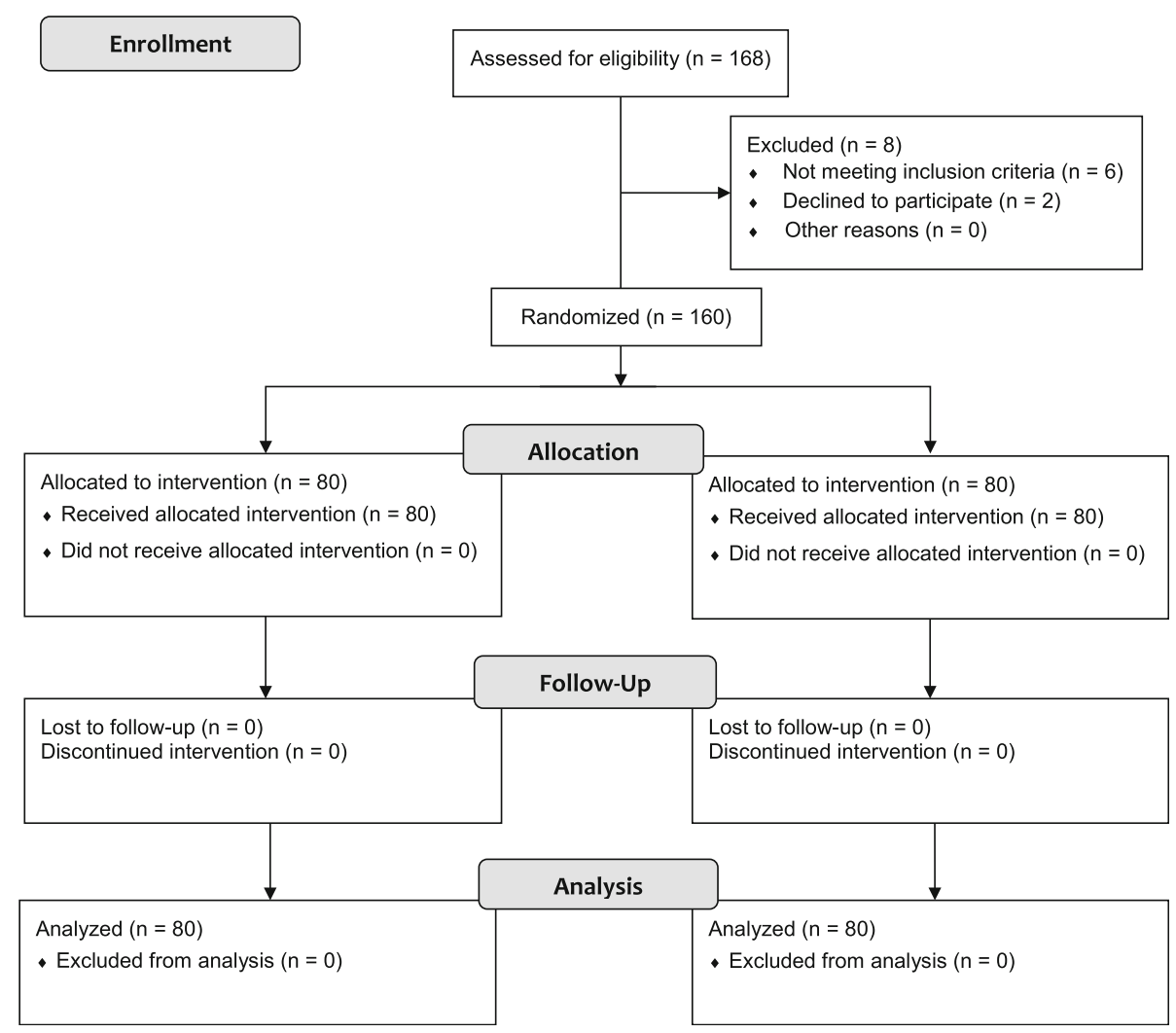

Fig. 1 Flowchart showing enrollment of patients

pressure was set at $120 \mathrm{mmHg}$, or $150 \mathrm{mmHg}$ above the last SBP measured just before tourniquet inflation (pre-inflation SBP). The surgeons were blinded to the allocation, and the inflation pressure was set by the circulating nurses who identified patient allocation from the concealed envelope. The cuff size was identical in all patients with $10 \mathrm{~cm}$ wide and $86 \mathrm{~cm}$ long.

All the operations were conducted by a single surgeon (senior author) with same manner. A midline longitudinal skin incision was used with followed medial parapatellar arthrotomy. One of the following two posterior stabilized implants were used according to the operator's discretion: Genesis II (Smith \& Nephew, Memphis, TN, USA), or e.motion PS PRO (Aesculap AG \& Co. KG., Tuttlingen, Germany). Patella was resurfaced routinely in all patients. All the implants were fixated with cement (Palacos ${ }^{\oplus}$ R, Heraeus Medical, Wehrheim, Germany). After the cement was completely polymerized, the tourniquet was deflated and arterial bleeding was controlled by electrocautery. A multimodal drug cocktail were injected in the periarticular tissue for the postoperative analgesia [19]. A vacuum-assisted drain was indwelled subcutaneously. Once the hemostasis and patellar tracking was confirmed, the tourniquet was reinflated and kept inflated until the completion of the dressing [20].

All patients were given intravenous patient-controlled analgesia (PCA). All the patients underwent thromboprophylaxis according to our pre-determined protocol, which is an individualized-approach strategy. Patients were assessed pre-operatively for the risk of pulmonary embolism (PE) or bleeding, then were classified into four risk-stratified categories, to tailor post-operative thromboprophylactic therapies. The proportion of risk-stratified groups for venous thromboembolism (VTE) prophylaxis did not differ between two groups. Rivaroxaban, $10 \mathrm{mg}$, was given orally, once a day for ten days, to patients with a standard risk for both PE and bleeding and to patients with an elevated risk for PE and a standard risk for bleeding. In patients with a standard risk for PE and an elevated risk for bleeding, an intermittent pneumatic compression (IPC) device was used for seven days. In patients with an elevated risk for both PE and bleeding, an IPC device was used for seven days along with aspirin $100 \mathrm{mg}$ orally once a day for six weeks. Continuous passive movement (CPM) 
Table 1 Demographic features and baseline data of study groups

\begin{tabular}{|c|c|c|c|}
\hline & $\begin{array}{l}\text { Conventional } \\
{[\mathrm{SBP}+150]} \\
(n=80)\end{array}$ & $\begin{array}{l}\text { Lower } \\
\text { Pressure } \\
{[\mathrm{SBP}+120]} \\
(n=80) \\
\end{array}$ & $P$ value \\
\hline Age (years) & $71.0(6.2)$ & $71.8(6.8)$ & 0.424 \\
\hline Sex: female & 73 (91\%) & $68(85 \%)$ & 0.222 \\
\hline Height (cm) & $152.4(6.3)$ & $152.8(7.8)$ & 0.784 \\
\hline Weight (kg) & $63.3(9.9)$ & $64.0(11.1)$ & 0.646 \\
\hline $\mathrm{BMI}\left(\mathrm{kg} / \mathrm{m}^{2}\right)$ & $27.1(3.3)$ & $27.4(4.0)$ & 0.647 \\
\hline Thigh circumference (cm) & $47.2(5.1)$ & $49.2(5.5)$ & 0.145 \\
\hline Hypertension & $55(70 \%)$ & $53(66 \%)$ & 0.647 \\
\hline \multicolumn{4}{|l|}{ Diagnosis } \\
\hline Primary osteoarthritis & 78 (97\%) & 76 (94\%) & 0.330 \\
\hline Secondary osteoarthritis & $00 \%)$ & $2(3 \%)$ & \\
\hline Inflammatory arthritis & $2(3 \%)$ & $3(3 \%)$ & \\
\hline Operated side: Right & $35(44 \%)$ & $42(53 \%)$ & 0.268 \\
\hline \multicolumn{4}{|l|}{ Operation type } \\
\hline Unilateral TKA & $38(48 \%)$ & $28(35 \%)$ & 0.261 \\
\hline Staged TKA: 1st & $22(28 \%)$ & $29(36 \%)$ & \\
\hline Staged TKA: 2nd & $20(25 \%)$ & $23(29 \%)$ & \\
\hline \multicolumn{4}{|l|}{ Implant } \\
\hline e-motion PS Pro ${ }^{\oplus}$ & 57 (71\%) & $64(80 \%)$ & 0.197 \\
\hline Genesis $\|^{\circledast}$ & $23(29 \%)$ & $16(20 \%)$ & \\
\hline \multicolumn{4}{|l|}{ Operation method } \\
\hline Conventional & $63(79 \%)$ & $68(85 \%)$ & 0.305 \\
\hline Navigation & $17(21 \%)$ & $12(15 \%)$ & \\
\hline Pre-inflation SBP (mmHg) & $105.1(15.9)$ & $111.2(14.8)$ & 0.012 \\
\hline $\begin{array}{l}\text { Initial tourniquet pressure } \\
(\mathrm{mmHg})\end{array}$ & $255.0(18.1)$ & $233.9(15.5)$ & $<0.001$ \\
\hline Tourniquet time (min) & $83.1(9.1)$ & $82.1(9.2)$ & 0.486 \\
\hline Operative time (min) & $99.3(16.0)$ & $99.5(15.7)$ & 0.925 \\
\hline $\begin{array}{l}\text { Maximum intraoperative SBP } \\
(\mathrm{mmHg})\end{array}$ & $124.1(16.8)$ & $121.4(15.1)$ & 0.278 \\
\hline Preoperative hemoglobin ( $\mathrm{g} / \mathrm{dL}$ ) & $12.4(1.96)$ & $12.1(2.1)$ & 0.406 \\
\hline
\end{tabular}

Data are presented as mean with the standard deviation in parentheses or number with the percentage in parentheses

$B M I$ body mass index, TKA total knee arthroplasty, SBP systolic blood pressure

and weight bearing with an assistive device were initiated beginning on day 2 and gradually increased over time. Patients undergoing unilateral TKA were discharged at seven days, and those who underwent staged bilateral TKA were discharged at 14 days (the 7 th postoperative day after the 2nd surgery).

\section{Outcome measurement}

The outcome measures included both intra-operative and post-operative parameters, which were evaluated within seven days after surgery. The primary efficacy outcome was the provision of a bloodless surgical field at the initiation of TKA (after skin incision, before arthrotomy). It was recorded as either present or absent. If the surgical field was obscured by blood oozing due to a sharp rise in SBP at any point after the initial exposure till the point of tourniquet deflation, it was recorded. The tourniquet pressure was increased additionally at this point by $30 \mathrm{mmHg}$ at a time, for a maximum of three times. The secondary efficacy outcomes were hemoglobin drop on postoperative 2nd and 5th day, drained amount and total blood loss, which was calculated using a formula based on patient blood volume and a decrease in hemoglobin that described in several previous studies [21-23]. The secondary safety outcome measures were pain in the thigh and knee, thigh complications, and other tourniquet-related complications. The pain levels were assessed on post-operative day 2 . Patients were questioned where is the most painful site among knee, thigh, or equal pain in the knee and thigh. In addition, pain level in the knee and thigh was evaluated by the patients using a visual analogue scale (VAS) from 0 to 10 , with 0 indicating no pain and 10 indicating the worst pain imaginable, and a 5-point Likert scale of increasing severity (no pain to extremely severe pain). Complications at the thigh were evaluated within seven postoperative days and recorded as ecchymosis, bullae, and skin necrosis. Ecchymosis was graded as follows: Grade 0 - none; Grade 1 - present but limited to the tourniquet site, $<3 \mathrm{~cm}$ wide; Grade $2->3 \mathrm{~cm}$ but still confined to the tourniquet site; Grade 3 - extending beyond the thigh. Patients were evaluated for other complications such as nerve palsy, symptomatic deep vein thrombosis (DVT), PE, and delayed rehabilitation defined as the inability to perform straight leg raise (SLR) for seven days after surgery.

\section{Statistical analysis}

Statistical analyses were conducted with SPSS $^{\circ}$ for Windows $^{\circ}$ (version 20.0, IBM, Chicago, IL, USA) and $p$ values $<0.05$ were considered significant for all comparisons. The initial achievement of a bloodless surgical field and the incidence of intraoperative blood oozing owing to a sharp SBP rise, hemoglobin drop, drained volume, calculated blood loss was compared between the two groups to reveal the efficacy of the lower tourniquet inflation pressure. The postoperative knee and thigh pain, thigh complications, and other tourniquet related complications were compared between the groups to confirm the safety of each method. Statistical significance was determined using the Chi square test or Fisher's exact test for categorical variables and the Student's t-test for continuous variables. 


\section{Results}

The pre-inflation SBP was lower in the conventional group, but the difference was only $6.1 \mathrm{mmHg}$ (105.1 vs. $111.2 \mathrm{mmHg}, p=0.012$ ) (Table 1). The initial tourniquet inflation pressure was significantly lower in the lower tourniquet pressure group than in the conventional group (255.0 vs. $233.9 \mathrm{mmHg} ; p<0.001$ ). However, the two groups did not differ with respect to tourniquet time, operative time, and the maximum intra-operative SBP.

The lower tourniquet pressure $(\mathrm{SBP}+120 \mathrm{mmHg})$ provided a bloodless surgical field comparable to that provided using conventional tourniquet pressure (SBP + $150 \mathrm{mmHg}$ ), and the blood loss was similar between the two groups (Table 2). The initial quality of the bloodless surgical field was good in both groups. A bloodless surgical field was achieved in 80 knees $(100 \%)$ in the conventional group and 79 cases $(99 \%)$ in the lower tourniquet pressure group $(p=1.000)$. Only one patient in the lower tourniquet pressure group did not have a bloodless surgical field at the initial exposure, which was addressed by increasing the tourniquet pressure by 30 $\mathrm{mmHg}$. One patient in the conventional group and two patients in the lower tourniquet pressure group had episodes of intraoperative blood oozing owing to a sharp increase in SBP, which obscured the initial bloodless surgical field. The maximum intraoperative SBP in the three patients was 136 [in the conventional group], 143 and $194 \mathrm{mmHg}$ [in the lower pressure group], with the pre-inflation SBP of 100, 110, and $140 \mathrm{mmHg}$, respectively. These patients required one instance of increase in the tourniquet pressure by $30 \mathrm{mmHg}$ and achieved adequate control of hemostasis. In addition, there was no difference in the hemoglobin drop on the 2nd and 5 th day after surgery, drained volume and the calculated blood loss (all $p>0.05$ ) (Table 1).

In terms of the safety outcomes, the two groups did not differ in any parameters, namely the pain level, thigh complications, or other tourniquet-related complications (Table 3). The VAS score and Likert scale for pain showed that there was no difference between the two groups regarding both the knee and thigh pain. The proportion of patients who suffered more severe pain in the thigh than in the knee did not differ between the groups. There was no difference in the frequency of the tourniquet-site ecchymosis between the two groups. One patient in each group developed bullae on the thigh. In addition, none of the patients developed thigh skin necrosis, nerve palsy, symptomatic DVT/PE, or delayed rehabilitation.

\section{Discussion}

The pneumatic tourniquet is widely used during TKA despite its use being a topic of debate [24, 25]. Considering the complications associated with higher tourniquet pressures, lower inflation pressures may theoretically lead to reduced post-operative pain levels and reduced incidence of tourniquet-related complications. We undertook this study to determine if the lower tourniquet inflation pressure would be as efficacious as the conventional inflation pressures and with a lower incidence of complications. We hypothesized that a lower tourniquet pressure has comparable efficacy to conventional inflation pressure, in terms of achieving a bloodless surgical field, and that it may lead to a decrease in tourniquet-related complications.

Our findings support our hypothesis that lower tourniquet pressure would provide a bloodless surgical field comparably to conventional inflation pressure. The rationale behind inflating the tourniquet beyond the SBP allowing a certain amount of safety margin is that it accounts for the intra-operative fluctuations in blood pressure and prevents oozing in the surgical field, which might obscure the surgical field. However, using a higher inflation pressure is one of the most important reasons for complications following tourniquet use $[3,26]$.

Table $\mathbf{2}$ Comparison of the efficacy of the tourniquet in both groups

\begin{tabular}{|c|c|c|c|}
\hline & $\begin{array}{l}\text { Conventional } \\
{[\mathrm{SBP}+150]} \\
(n=80)\end{array}$ & $\begin{array}{l}\text { Lower Pressure } \\
{[\mathrm{SBP}+120]} \\
(n=80)\end{array}$ & $P$ value \\
\hline Bloodless surgical field (initial) & $80 / 80(100 \%)$ & $79 / 80(99 \%)$ & 1.000 \\
\hline Intra-operative blood oozing after sharp rise in SBP & $1 / 80(1 \%)$ & $2^{*} / 80(3 \%)$ & 1.000 \\
\hline Total failure of providing bloodless surgical field & $1 / 80(1 \%)$ & $2^{*} / 80(3 \%)$ & 1.000 \\
\hline Hemoglobin drop on 2nd day & $2.7(1.1)$ & $2.4(1.3)$ & 0.184 \\
\hline Hemoglobin drop on 5th day & $2.8(1.3)$ & $2.4(1.8)$ & 0.076 \\
\hline Drained volume $(\mathrm{ml})$ & $23(35)$ & $27(47)$ & 0.482 \\
\hline Calculated blood loss (ml) & $744(256)$ & $708(283)$ & 0.404 \\
\hline
\end{tabular}

Data are presented as a number of patients with the percentage or mean values with the standard deviation in the parenthesis

* One patient in the lower tourniquet pressure group failed to achieve a bloodless surgical field initially and showed intraoperative blood oozing after a sharp rise in SBP

SBP systolic blood pressure 
Table 3 Comparison of safety parameters among groups

\begin{tabular}{|c|c|c|c|}
\hline & $\begin{array}{l}\text { Conventional } \\
{[\mathrm{SBP}+150]} \\
(n=80)\end{array}$ & $\begin{array}{l}\text { Lower Pressure } \\
{[\mathrm{SBP}+120]} \\
(n=80)\end{array}$ & $P$ value \\
\hline \multicolumn{4}{|l|}{ More painful site } \\
\hline Knee & $59(74 \%)$ & $60(75 \%)$ & \multirow[t]{3}{*}{0.731} \\
\hline Thigh & 15 (19\%) & $12(15 \%)$ & \\
\hline Knee $=$ Thigh & $6(7 \%)$ & $8(10 \%)$ & \\
\hline Knee pain (VAS) & $2.8(1.4)$ & $3.0(1.6)$ & 0.451 \\
\hline \multicolumn{4}{|l|}{ Knee pain (Likert) } \\
\hline No or Slight & 35 (44\%) & 37 (46\%) & \multirow[t]{2}{*}{0.751} \\
\hline$\geq$ Moderate & 45 (56\%) & $43(54 \%)$ & \\
\hline Thigh pain (VAS) & $1.5(1.7)$ & $1.3(1.6)$ & 0.270 \\
\hline \multicolumn{4}{|l|}{ Thigh pain (Likert) } \\
\hline No or Slight & $61(76 \%)$ & $64(80 \%)$ & \multirow[t]{2}{*}{0.566} \\
\hline$\geq$ Moderate & $19(24 \%)$ & $16(20 \%)$ & \\
\hline \multicolumn{4}{|l|}{ Thigh ecchymosis } \\
\hline Grade $0-1$ & 77 (96\%) & 73 (91\%) & \multirow[t]{2}{*}{0.191} \\
\hline Grade $2-3$ & $3(4 \%)$ & 7 (9\%) & \\
\hline Thigh bullae & $1(1 \%)$ & $1(1 \%)$ & 1.000 \\
\hline Thigh necrosis & $0(0 \%)$ & $0(0 \%)$ & N/A \\
\hline Nerve palsy & $0(0 \%)$ & $0(0 \%)$ & N/A \\
\hline Symptomatic DVT & $0(0 \%)$ & $0(0 \%)$ & $\mathrm{N} / \mathrm{A}$ \\
\hline Symptomatic PE & $0(0 \%)$ & $0(0 \%)$ & N/A \\
\hline Delayed rehabilitation & $0(0 \%)$ & $0(0 \%)$ & $\mathrm{N} / \mathrm{A}$ \\
\hline
\end{tabular}

Data are presented as a number of patients and the percentage, except for the knee and thigh pain (VAS), the data for which are presented as mean and standard deviation

VAS visual analog scale, DVT deep vein thrombosis, PE pulmonary embolism

Hence, use of the lowest possible inflation pressure is recommended, provided the tourniquet inflation pressure is sufficient to provide a bloodless surgical field. The mean pressure in the lower tourniquet pressure group (SBP + $120 \mathrm{mmHg}$ ) was $234 \mathrm{mmHg}$, which is considerably lower than the conventional group (SBP $+150 \mathrm{mmHg}$ ) or the fixed pressure of 300 or $350 \mathrm{mmHg}$ used in other studies [6-8]. Additionally, only two patients in the lower tourniquet pressure group had an inadequate tourniquet pressure, with the incidence of tourniquet failure similar to that of a previous study [4]. This failure to achieve a bloodless surgical field was addressed by increasing the tourniquet pressure by $30 \mathrm{mmHg}$. Previous prospective randomized controlled studies that compared the lower tourniquet pressure with the higher tourniquet pressure reported that, the lower tourniquet pressure could provide a sufficient bloodless surgical field that is comparable to that provided by the higher tourniquet pressure (Table 4) $[4,12,14-16,27]$. The mean inflation pressures in the lower inflation pressure groups were in the 223-260 $\mathrm{mmHg}$ range, which is similar to our result $(234 \mathrm{mmHg})$, except for the mean initial inflation pressure of 182
$\mathrm{mmHg}$ in a study by Tuncali et al., which used arterial occlusion pressure (AOP) estimation method [27]. In addition, a previous study reported an inflation pressure of $250 \mathrm{mmHg}$ as being adequate for lower extremity surgery [28], and another study found that a mean inflation pressure of $231 \mathrm{mmHg}$ was adequate for the provision of a bloodless field [29]. The use of limb occlusion pressure (LOP) estimation can help individualize and decrease tourniquet inflation pressures, and modern tourniquet systems permit an automated estimation of LOP through a probe incorporated in the tourniquet system itself [4]. However, these devices may not be available at every institution, and LOP measurement involves performing an additional procedure and more time. An AOP estimation method was introduced which reported to be more simple than LOP estimation method, but this method also require measurement of extremity circumference to use estimation equation [27, 30]. Our method of adding 120 $\mathrm{mmHg}$ to the SBP is simple and practical in its application and has comparable results to those with conventional tourniquet inflation pressures. The efficacy of the lower tourniquet pressure, $\mathrm{SBP}+120 \mathrm{mmHg}$, is also supported by the no difference result in the hemoglobin drop, drained volume and calculated blood loss between the two groups.

Contrary to our expectations, using the lower tourniquet pressure did not decrease the incidence of tourniquet-related complications. However, the incidence of complications in both the study groups was rare. This may be related to the lower tourniquet pressure as well as lower ischemia time. The mean ischemia time in both our study groups was less than $100 \mathrm{~min}$. A tourniquet time more than $100 \mathrm{~min}$ has been shown to increase complications [9]. Additionally, lower tourniquet pressures have been shown to decrease the incidence of complications following tourniquet use $[4,31]$. A probable reason for the lack of difference in the incidence of complications among the two groups in our study is that our study was underpowered to detect serious complications following tourniquet use, which have a low incidence rate of one in 4200 [32]. Similarly to our findings, a study comparing lower tourniquet pressures to conventional inflation pressures (mean $252 \mathrm{mmHg}$ ) found no difference in post-operative pain levels [4]. However, lesser pain levels have been reported in the lower tourniquet pressure groups compared to conventional (300 or $350 \mathrm{mmHg}$ ) pressure groups $[14,33]$. This might stem from the extent of the difference in the inflation pressures of the lower and conventional pressure groups in each study. The inflation pressure differences between the lower and conventional pressure groups in the two studies, which reported different pain levels between the groups, were 120 and $135 \mathrm{mmHg}$ of mean tourniquet pressure, respectively. In the current study, the pressure difference was 


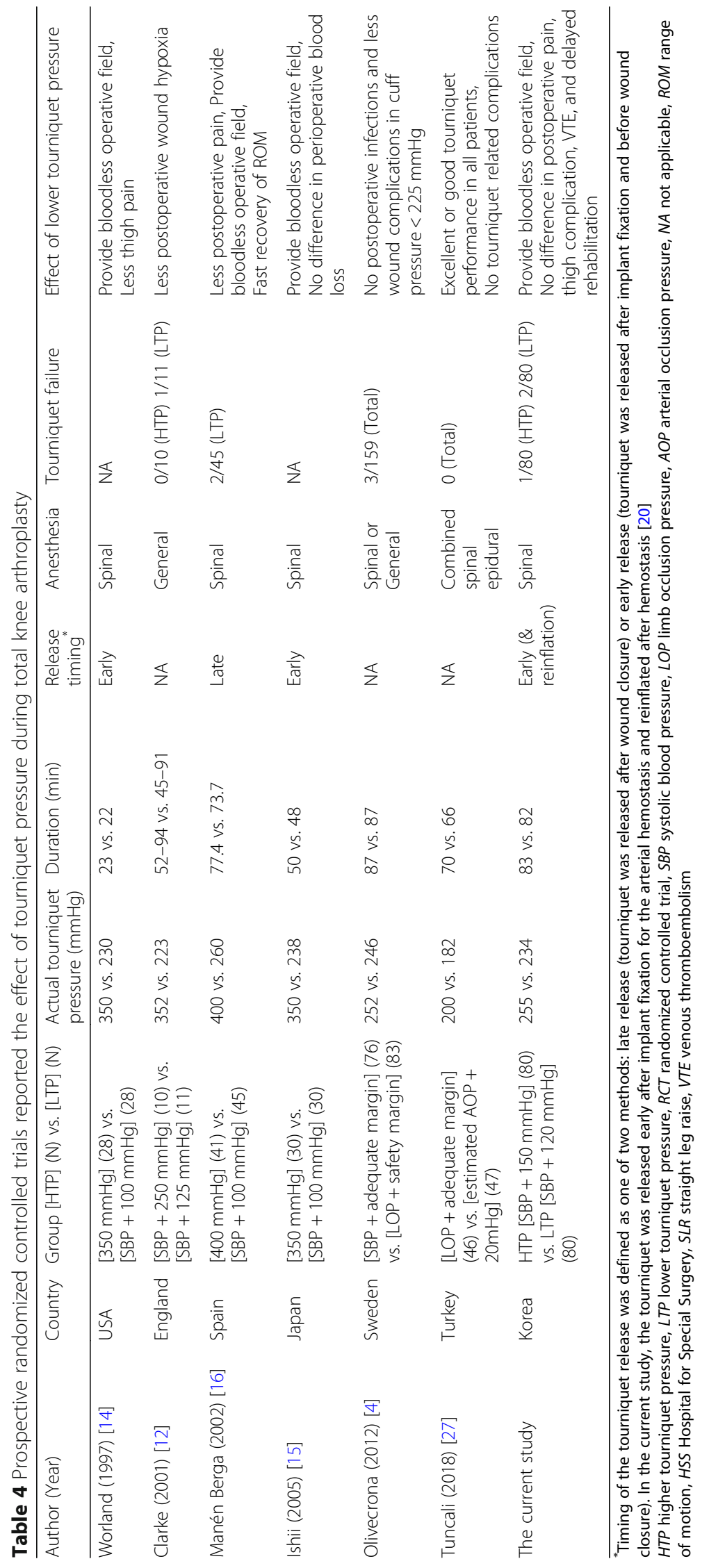


only $21 \mathrm{mmHg}$. One patient in each of our study groups developed skin bullae. This was probably due to frictional burns caused by an ill-fitted tourniquet. We did not experience any major complications such as thigh bullae or necrosis, nerve palsy, symptomatic DVT/PE, or delayed rehabilitation. This finding supports both methods of determining the tourniquet pressure as safely applicable in clinical practice, even when using the conventional method of $150 \mathrm{mmHg}$ above the SBP.

Our study has the following several limitations: First, the majority of patients in both the groups were women. However, those selected were consecutive patients of our institute, typically showing about $90 \%$ of female predominance. This female predominance of patients undergoing TKA in the Korean population is well documented [34, 35]. Second, uniform thrombo-prophylaxis was not given to all the participants, but a risk-stratified individualized approach was used following the routine protocol of the authors' institute. However, we think that this issue did not seriously skew the results, because all patients were randomly allocated regardless of their risk for PE and bleeding. Actually, none of the patients suffered symptomatic DVT or PE. We did not expect a considerable difference among the two groups concerning blood loss, as has been documented previously [15]. Third, the quality of the surgical field was evaluated in a subjective manner relying on the operator's decision. However, it is difficult to rate the quality of the bloodless surgical field objectively; therefore, previous studies also employed a subjective evaluation method [4, 17, 29]. Olivecrona et al. used VAS graded by the operator after surgery regarding the quality of the bloodless surgical field and the technical difficulty caused by the quality of it [4]. Ishii et al. rated the quality of the bloodless field as poor, fair, good, or excellent, and noted any changes in the quality of the surgical field throughout the operation [17]. In the report by Reid et al., hemostasis was rated by the operating surgeon as good, adequate or failed during operation [29]. We only considered the blood oozing that definitely interfere with the surgical procedure as a failure of achieving bloodless surgical field, rather than rating into several categories or rating using visual analog scale, to minimize the effect of the subjective evaluation. In addition, there was no difference in the bleeding-related parameters, such as hemoglobin drop, drained volume, and calculated blood loss, which indirectly support the equivalent bloodless surgical field quality of the two groups. Fourth, the dosage of the spinal anesthesia was not identical to all the patients but was modified based on the body weight, which was entirely determined by the anesthesiologist. The amount of anesthetic drug may affect the pre-inflation SBP. However, there was no difference in the body weight and the BMI between the two groups, the difference of the dosage may not affect the result significantly. Fifth, there was significant difference in the pre-inflation pressure between the two groups, although the patients were randomly allocated. The difference of the pre-inflation pressure affected the difference of the initial inflation pressure, which was determined based on the pre-inflation SBP: About $21 \mathrm{mmHg}$ of difference was noted, rather than mathematically anticipated $30 \mathrm{mmHg}$ of difference between the two methods. Sixth, clinical significance of this study may be limited, as previous studies reported even lower tourniquet pressure method, SBP plus $100 \mathrm{mmHg}$, rather than SBP $+120 \mathrm{mmHg}$ of this study [14-16]. However, considering the high failure in achieving bloodless surgical field in a study which used SBP $+100 \mathrm{mmHg}$ [17], our study add an evidence of using the SBP $+120 \mathrm{mmHg}$ as an alternative method.

\section{Conclusion}

The use of lower tourniquet inflation pressure, 120 $\mathrm{mmHg}$ above the SBP, successfully provides a bloodless field comparable to that provided by the conventionally used higher pressure of $150 \mathrm{mmHg}$ above the SBP, with a similar incidence of complications. Therefore, we recommend using a tourniquet inflation pressure of 120 $\mathrm{mmHg}$ above the SBP during TKA.

\section{Abbreviations \\ CPM: Continuous passive movement; DVG: Deep vein thrombosis; IPC: Intermittent pneumatic compression; LOP: Limb occlusion pressure; PCA: Patient-controlled analgesia; PE: Pulmonary embolism; SBP: Systolic blood pressure; SLR: Straight leg raise; TKA: Total knee arthroplasty; VAS: Visual analogue scale; VTE: Venous thromboembolism}

\section{Acknowledgments}

Not applicable.

Funding

No funding was used for this study.

\section{Availability of data and materials}

The datasets generated and/or analyzed during the current study are not publicly available but are available from the corresponding author on reasonable request.

\section{Authors' contributions \\ TKK conceived of the study and participated in its design and coordination and contributed to drafting the manuscript. ABB substantially contributed to drafting the manuscript. JAS contributed significantly in revising the manuscript. JHP analyzed and interpreted the data and participated in the manuscript writing. YGN conceived and designed the study, collected the data, and was a major contributor in writing the manuscript. All authors read and approved the final manuscript.}

\section{Authors' information}

One of the authors (TKK) received research funding from Smith \& Nephew and B. Braun Aesculap, and is a design consultant for Smith \& Nephew and B. Braun Aesculap. None of the other authors (ABB, JAS, JHP, YGN) have relevant conflicts of interest to declare.

Each author certifies that his or her institution has approved the human protocol for this.

Investigation, that all investigations were conducted in conformity with ethical principles of research, and that informed consent was obtained. 


\section{Ethics approval and consent to participate}

All individuals provided informed, written consent to participate, and the protocol was approved by Seoul National University Hospital Institutional Review Board (IRB No: B-1310-221-007) in accordance with the guidelines established by the Declaration of Helsinki for research involving human participants.

\section{Consent for publication}

Not applicable.

\section{Competing interests}

One of the authors (TKK) received research funding from Smith \& Nephew and B. Braun Aesculap, and is a design consultant for Smith \& Nephew and B. Braun Aesculap. None of the other authors (ABB, JAS, JHP, YGN) have relevant conflicts of interest to declare.

\section{Publisher's Note}

Springer Nature remains neutral with regard to jurisdictional claims in published maps and institutional affiliations.

\section{Author details}

'TK Orthopedic Surgery, Seongnam-si, Gyeonggi-do, Republic of Korea. ${ }^{2}$ Pioneer Hospital, New Panvel, Navi Mumbai, Maharashtra, India. ${ }^{3}$ Department of Orthopedic Surgery, Gachon University Gil Medical Center, Incheon, Republic of Korea. ${ }^{4}$ Department of Orthopedic Surgery, CM Hospital, 13, Yeongdeungpo-ro 36-gil, Yeongdeungpo-gu, Seoul 07301, Republic of Korea. ${ }^{5}$ Former affiliation: Gachon University Gil Medical Center, Incheon, Republic of Korea.

\section{Received: 3 October 2018 Accepted: 16 May 2019}

\section{Published online: 04 June 2019}

\section{References}

1. Fukuda A, Hasegawa M, Kato K, Shi D, Sudo A, Uchida A. Effect of tourniquet application on deep vein thrombosis after total knee arthroplasty. Arch Orthop Trauma Surg. 2007;127(8):671-5.

2. Smith $\mathrm{TO}$, Hing $\mathrm{CB}$. Is a tourniquet beneficial in total knee replacement surgery? A meta-analysis and systematic review. Knee. 2010;17(2):141-7.

3. Noordin S, McEwen JA, Kragh JF Jr, Eisen A, Masri BA. Surgical tourniquets in orthopaedics. J Bone Joint Surg Am. 2009;91(12):2958-67.

4. Olivecrona C, Ponzer S, Hamberg P, Blomfeldt R. Lower tourniquet cuff pressure reduces postoperative wound complications after total knee arthroplasty: a randomized controlled study of 164 patients. J Bone Joint Surg Am. 2012;94(24):2216-21.

5. Tejwani NC, Immerman I, Achan P, Egol KA, McLaurin T. Tourniquet cuff pressure: the gulf between science and practice. J Trauma. 2006;61(6):1415-8,

6. Vandenbussche E, Duranthon LD, Couturier M, Pidhorz L, Augereau B. The effect of tourniquet use in total knee arthroplasty. Int Orthop. 2002;26(5):306-9.

7. Mittal R, Ko V, Adie S, Naylor J, Dave J, Dave C, Harris IA, Hackett D, Ngo D, Dietsch $\mathrm{S}$. Tourniquet application only during cement fixation in total knee arthroplasty: a double-blind, randomized controlled trial. ANZ J Surg. 2012;82(6):428-33.

8. Bould M, Freeman BJ, Pullyblank A, Newman JH. Blood loss in sequential bilateral total knee arthroplasty. J Arthroplast. 1998;13(1):77-9.

9. Olivecrona C, Lapidus $L$, Benson L, Blomfeldt R. Tourniquet time affects postoperative complications after knee arthroplasty. Int Orthop. 2013;37(5):827-32.

10. Wakankar HM, Nicholl JE, Koka R, D'Arcy JC. The tourniquet in total knee arthroplasty. A prospective, randomised study. J Bone Joint Surg Br. 1999:81(1):30-3.

11. Barwell J, Anderson G, Hassan A, Rawlings I. The effects of early tourniquet release during total knee arthroplasty: a prospective randomized doubleblind study. J Bone Joint Surg Br. 1997;79(2):265-8.

12. Clarke MT, Longstaff $L$, Edwards D, Rushton N. Tourniquet-induced wound hypoxia after total knee replacement. J Bone Joint Surg Br. 2001;83(1):40-4.

13. Tetro AM, Rudan JF. The effects of a pneumatic tourniquet on blood loss in total knee arthroplasty. Can J Surg. 2001;44(1):33-8.

14. Worland RL, Arredondo J, Angles F, Lopez-Jimenez F, Jessup DE. Thigh pain following tourniquet application in simultaneous bilateral total knee replacement arthroplasty. J Arthroplast. 1997;12(8):848-52.
15. Ishii Y, Matsuda Y. Effect of tourniquet pressure on perioperative blood loss associated with cementless total knee arthroplasty: a prospective, randomized study. J Arthroplast. 2005;20(3):325-30.

16. Manen Berga F, Novellas Canosa M, Angles Crespo F, Bernal Dzekonski J. Effect of ischemic tourniquet pressure on the intensity of postoperative pain. Revista espanola de anestesiologia y reanimacion. 2002;49(3):131-5.

17. Ishii Y, Noguchi H, Matsuda Y, Takeda M, Higashihara T. A new tourniquet system that determines pressures in synchrony with systolic blood pressure in total knee arthroplasty. J Arthroplast. 2008;23(7):1050-6.

18. Olivecrona C, Tidermark J, Hamberg P, Ponzer S, Cederfjall C. Skin protection underneath the pneumatic tourniquet during total knee arthroplasty: a randomized controlled trial of 92 patients. Acta Orthop. 2006;77(3):519-23.

19. Koh IJ, Kang YG, Chang CB, Do SH, Seong SC, Kim TK. Does periarticular injection have additional pain relieving effects during contemporary multimodal pain control protocols for TKA?: a randomised, controlled study. Knee. 2012;19(4):253-9.

20. Na YG, Bamne AB, Won HH, Kim TK: After early release of tourniquet in total knee arthroplasty, should it be reinflated or kept deflated? A randomized trial. Knee surgery, sports traumatology, arthroscopy : official journal of the ESSKA 2015.

21. Nadler SB, Hidalgo JH, Bloch T. Prediction of blood volume in normal human adults. Surgery. 1962;51(2):224-32.

22. Good L, Peterson E, Lisander B. Tranexamic acid decreases external blood loss but not hidden blood loss in total knee replacement. Br J Anaesth. 2003;90(5):596-9.

23. Konig G, Hamlin BR, Waters JH. Topical tranexamic acid reduces blood loss and transfusion rates in total hip and total knee arthroplasty. J Arthroplast. 2013;28(9):1473-6.

24. Lee GC, Hawes T, Cushner FD, Scott WN. Current trends in blood conservation in total knee arthroplasty. Clin Orthop Relat Res. 2005;440:170-4.

25. Jaschinski T, Pieper D, Eikermann M, Steinhausen S, Linke C, Heitmann T, Pani M, Neugebauer E. Current status of total hip and knee replacements in Germany - results of a nation-wide survey. Zeitschrift fur Orthopadie und Unfallchirurgie. 2014;152(5):455-61.

26. Klenerman L. Tourniquet time--how long? Hand. 1980;12(3):231-4.

27. Tuncali B, Boya H, Kayhan Z, Arac S. Tourniquet pressure settings based on limb occlusion pressure determination or arterial occlusion pressure estimation in total knee arthroplasty? A prospective, randomized, double blind trial. Acta Orthop Traumatol Turc. 2018;52(4):256-60.

28. Van Roekel HE, Thurston AJ. Tourniquet pressure: the effect of limb circumference and systolic blood pressure. J Hand Surg Br. 1985;10(2):142-4.

29. Reid HS, Camp RA, Jacob WH. Tourniquet hemostasis. A clinical study. Clin Orthop Relat Res. 1983(177):230-4.

30. Tuncali B, Karci A, Tuncali BE, Mavioglu O, Ozkan M, Bacakoglu AK, Baydur H, Ekin A, Elar Z. A new method for estimating arterial occlusion pressure in optimizing pneumatic tourniquet inflation pressure. Anesth Analg. 2006;102(6):1752-7.

31. Olivecrona C, Blomfeldt R, Ponzer S, Stanford BR, Nilsson BY. Tourniquet cuff pressure and nerve injury in knee arthroplasty in a bloodless field: a neurophysiological study. Acta Orthop. 2013;84(2):159-64.

32. Odinsson A, Finsen V. Tourniquet use and its complications in Norway. J Bone Joint Surg Br. 2006;88(8):1090-2.

33. Unver B, Karatosun V, Tuncali B. Effects of tourniquet pressure on rehabilitation outcomes in patients undergoing total knee arthroplasty. Orthop Nurs. 2013;32(4):217-22.

34. Cho HJ, Chang CB, Kim KW, Park JH, Yoo JH, Koh IJ, Kim TK. Gender and prevalence of knee osteoarthritis types in elderly Koreans. J Arthroplast. 2011;26(7):994-9.

35. Koh IJ, Kim TK, Chang CB, Cho HJ, In Y. Trends in use of total knee arthroplasty in Korea from 2001 to 2010. Clin Orthop Relat Res. 2013:471(5):1441-50 